\title{
Globally covering a-priori regional gravity covariance models
}

\author{
D. Arabelos ${ }^{1}$ and C. C. Tscherning ${ }^{2}$ \\ ${ }^{1}$ Dept. of Geodesy and Surveying, Aristotle University of Thessaloniki, Univ. Box 474, GR-54 124 Thessaloniki, Greece \\ ${ }^{2}$ Dept. of Geophysics, University of Copenhagen, Juliane Maries Vej 30, DK-2100 Copenhagen, Denmark
}

\begin{abstract}
Gravity anomaly data generated using Wenzel's GPM98A model complete to degree 1800, from which OSU91A has been subtracted, have been used to estimate covariance functions for a set of globally covering equal-area blocks of size $22.5^{\circ} \times 22.5^{\circ}$ at Equator, having a $2.5^{\circ}$ overlap. For each block an analytic covariance function model was determined. The models are based on 4 parameters: the depth to the Bjerhammar sphere (determines correlation), the free-air gravity anomaly variance, a scale factor of the OSU91A error degree-variances and a maximal summation index, $N$, of the error degree-variances. The depth of Bjerhammar-sphere varies from $-134 \mathrm{~km}$ to nearly zero, $N$ varies from 360 to 40 , the scale factor from 0.03 to 38.0 and the gravity variance from 1081 to $24\left(10 \mu \mathrm{ms}^{-2}\right)^{2}$. The parameters are interpreted in terms of the quality of the data used to construct OSU91A and GPM98A and general conditions such as the occurrence of mountain chains. The variation of the parameters show that it is necessary to use regional covariance models in order to obtain a realistic signal to noise ratio in global applications.
\end{abstract}

Key words. GOCE mission, Covariance function, Spacewise approach

\section{Introduction}

When using the so-called space-wise approach for the modelling of GOCE data (ESA,SP-1233, 1999) it has been proposed to construct a global gravity field model based on regionally interpolated data such as $T_{z z}$ on a sphere with a radius equal to the mean distance of GOCE from the Earths centre (Sansó and Tscherning, 2002). Furthermore it is planned to screen the GOCE data from gross errors by predicting the observations from close, earlier observed, data (Arabelos and Tscherning, 1998). Least-Squares Collocation may be used for both tasks, but it requires that the regional

Correspondence to: D. Arabelos (arab@eng.auth.gr) covariance functions are estimated and subsequently modelled analytically.

The size of the regions will depend on the number of data which it is reasonable to handle in on LSC estimation computation. At present 50000 observations give a turn-around time of a day using a $2 \mathrm{GHz}$ PC. At the time of the expected launch of GOCE (2005+), it will certainly not be a problem to handle 200000 observations simultaneously. Using 4 independent observations per "normal"-point spaced $4 \mathrm{~s}$ apart (it is the time difference along the orbit), leads to a block size of approximately $20^{\circ}$. Considering the spatial correlation both of the errors and the signal, a minimum overlap of $1^{\circ}$ is needed. We have therefore initially chosen equal area blocks of size $22.5^{\circ}$ as our "regions".

For the estimation of the empirical covariance functions (by forming sums of products) we generated data using the GPM98A model (Wenzel , 1998) complete to degreee 1800, see Sect. 2. The analytic covariance functions were modeled as described in Sect. 3 using one of the models proposed by Tscherning and Rapp (1974). These models are simpler than other models (Jekeli, 1978), but have through many years of use shown to be surprisingly well suited for analytic covariance modeling.

The most interesting results are the general consistency of the parameters defining the analytic models. There are however, some anomalies the occurrence of which we have tried to explain.

\section{The gravity field}

For the representation of the gravity field in a global scale, the geopotential model GPM98A was used, complete to degree and order $N=1800$. Then the contribution of OSU91A to degree $N=360$ was subtracted. We have used OSU91A instead EGM96, in order to do our experiment more realistic, since the last model has been treated as a "start model" in the computation of GPM98A (Wenzel , 1998). In this way, our residual field has some remaining low-frequency compo- 
nents, like it happens when the contribution of a gepotential model is subtracted from observed gravity data. It should be noted that real data instead of band-limited data up to degree 1800 may involve higher frequencies. However, higher frequencies have a small impact on the estimation of the parameters of the covariance functions.

For the computation of the regional covariance models the area of the Earth was divided in 9 zones, having a constant $\delta \varphi=20^{\circ}$. Each zone was divided in a number of blocks of varying $\delta \lambda$, so that a number of $10820^{\circ}$ (almost) equalarea blocks were formed. Each block had an overlap of $2.5^{\circ}$ with the neighboring blocks (i.e. the actual area covered by a block at the equator was $22.5^{\circ} \times 22.5^{\circ}$ ). For all blocks free-air gravity anomalies were computed on grids having constant $\delta \varphi=0.1^{\circ}$ and varying $\delta \lambda$, depending on the latitude. The size of each block and the equidistance of the corresponding grid are shown in the headings of (Table 1).

\section{Covariance models}

For each of the 108 equal-area blocks the empirical covariance function was computed. The fitting of the empirical covariance functions to analytical models was performed using the well known formula

$$
\begin{aligned}
C(P, Q) & =\sum_{i=2}^{N} \hat{\sigma}_{i}\left(\frac{R_{B}^{2}}{r r^{\prime}}\right)^{i+2} P_{i}\left(\cos \psi_{P Q}\right) \\
& +\sum_{i=N+1}^{\infty} \frac{A(i-1)}{(i-2)(i+24)}\left(\frac{R_{B}^{2}}{r r^{\prime}}\right)^{i+2} P_{i}\left(\cos \psi_{P Q}\right)
\end{aligned}
$$

In Eq. (1) $N$ is the degree of the expansion of the geopotential model used for the reduction of gravity anomalies, $r, r^{\prime}$ are the distances of the points $P, Q$ from the Earth's center, $\hat{\sigma}_{i}$ error anomaly degree variances in units $\left(10 \mu \mathrm{ms}^{-2}\right)^{2}$ associated with the OSU91A coefficients, $R_{B}$ the radius of the so-called Bjerhammar sphere, $P_{i}$ the Legendre polynomial of degree $i, \psi_{P Q}$ the spherical distance between $P$ and $Q$, while $A$ is a free parameter in units of $\left(10 \mu \mathrm{ms}^{-2}\right)^{2}$ (i.e. the gravity anomaly variance).

Since the error degree variances of a geopotential model reflect the behavior of this model globally, in regional or local computations it is necessary to multiply the error degree variances by a scale factor $f$. This scale factor has to be defined through the fitting procedure. In this way, the free parameters to be estimated in Eq. (1) are $A, R_{B}$ and $f$. Instead of $R_{B}$, the difference $R_{E}-R_{B}$ is usually computed, where $R_{E}$ is the mean radius of the Earth.

For the computations the FORTRAN program "COVFIT" (Tscherning et al., 1994) was used. The quality of the fitting was expressed in terms of the RMS of the differences between empirical and model values, divided by the corresponding errors, associated with each of the estimated covariance values. These errors are computed taking into account the variances and the area specifications, according to

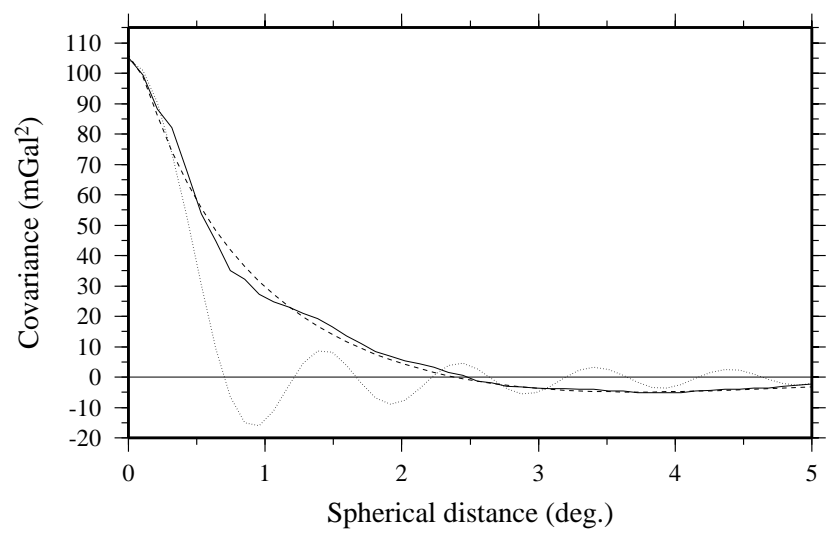

Fig. 1. Worst fit of the empirical (solid line) to the model (dashed line) covariance function

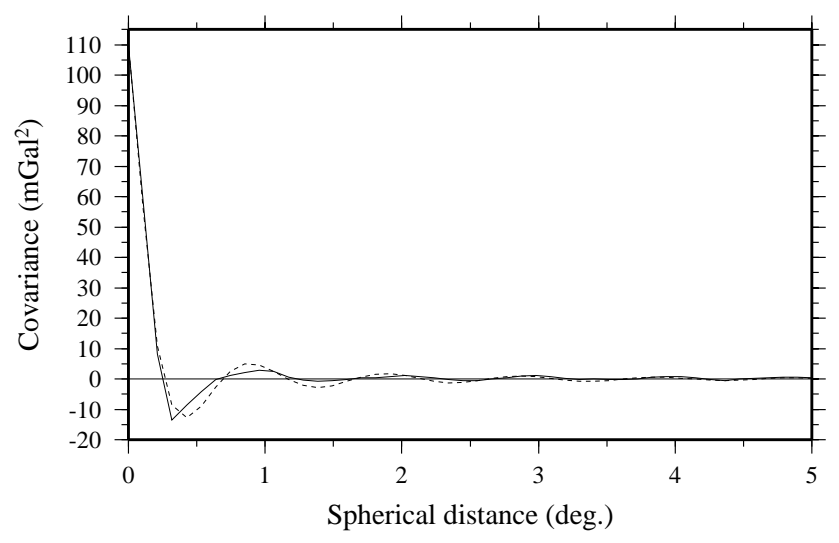

Fig. 2. Very good fit of the empirical (solid line) to the model (dashed line) covariance function.

Knudsen (1987):

$\operatorname{err}_{k}=\frac{C}{\sqrt{n_{0}}} \frac{n_{k}}{N_{k}}$

where $C=\sqrt{C_{0} C_{0}^{\prime}}$ is the geometric mean of the variances $C_{0}, C_{0}^{\prime}$ of the observations $y$ and $y^{\prime}$ respectively, $n_{0}$ is the number of cells covering the area, $N_{k}$ is the actual number of products and $n_{k}$ is the expected number of products. In our case is $y=y^{\prime}$.

$n_{k}$ depends on the size of the cells, the size of the area, the spherical sistance and the size of the interval.

$n_{k}=$

$\left\{\begin{array}{l}\left(\varphi_{2}-\varphi_{1}\right) / \Delta \varphi\left(\lambda_{2}-\lambda_{1}\right) / \Delta \lambda \text { for } k=0 \\ 2 \pi n_{0}\left(\psi_{k}+\psi_{k-1}\right) / 2\left(\psi_{k}-\psi_{k-1}\right) /(\Delta \varphi \Delta \lambda) \text { for } k>0\end{array}\right\}$.

The estimated parameters for each block vary within wide limits: The gravity variance varies from 1081 to $24\left(10 \mu \mathrm{ms}^{-2}\right)^{2}$, the depth to the Bjerhammar sphere from almost zero to $-134 \mathrm{~km}$, and the scale factor from 0.03 to 38 . 

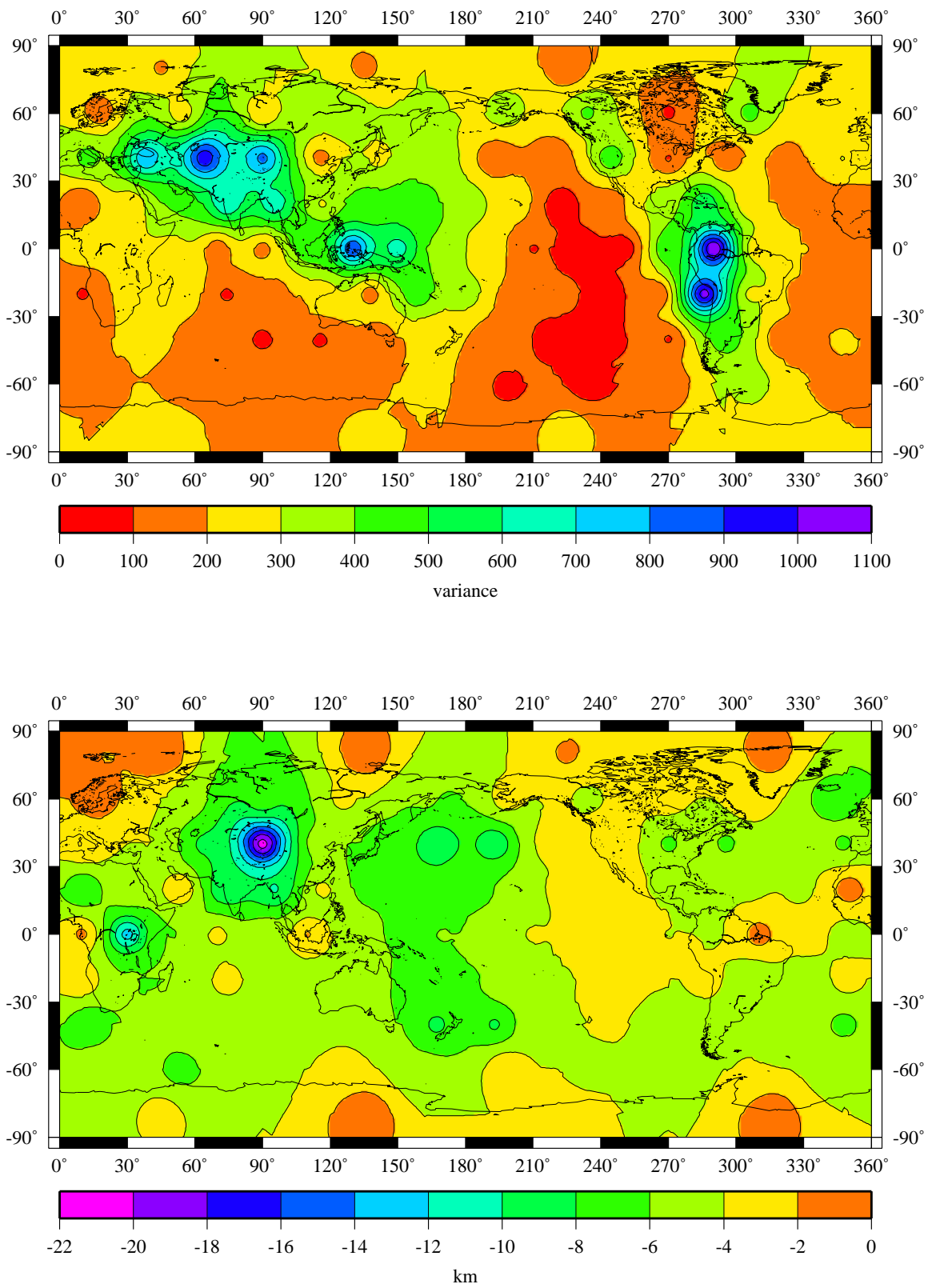

Fig. 3. Variances of the covariance models of the $20^{\circ}$ equal area blocks.

Fig. 4. Depths to the Bjerhammar sphere of the covariance models of the $20^{\circ}$ equal area blocks.
Furthermore, the quality of the fitting is very poor in some blocks, where the RMS takes unexpected large values (up to 72). To overcome this problem we have added one more free parameter in the fitting procedure, namely the maximum summation index $N$ of the error degree variances. The FORTRAN program "COVFIT" was modified accordingly. The estimated values on $N$ varies from 360 to 40 . Simultaneously, a considerable decrease of the RMS and of the depth to the Bjerhammar sphere was observed, with a corresponding increase of the scale factor. The changes in the variance could be considered negligible. Considerable improvement was observed in the cases of a serious decrease of $N$. In Table 1 one example per zone is shown, presenting the better result in RMS, achieved when $N$ was treated as a free parameter. In parenthesis the RMS with fixed $N$ (equal to 360) is shown. The corresponding decrease of $N$ is remarkable. However, there are 39 cases without change of $N$.

Figure 1 shows a very worst fit of the empirical (solid line) to the model (dashed line) covariance function concerning block 29, with $N$ fixed to 360 . The RMS was equal to 19.480. The situation was improved (long dashes), when $N$ was treated as an additional free parameter. In this case the RMS was dropped to 2.717. On the other hand, Fig. 2 shows an example of a very good fit, concerning block 80 . In this case, the RMS was equal to 1.594 and no changes of the other parameters were observed when $N$ was used as a free parameter.

The interpretation of the results of the computations is a difficult task, since they are affected by several parameters. Generally speaking in oceanic blocks, where homogeneous 


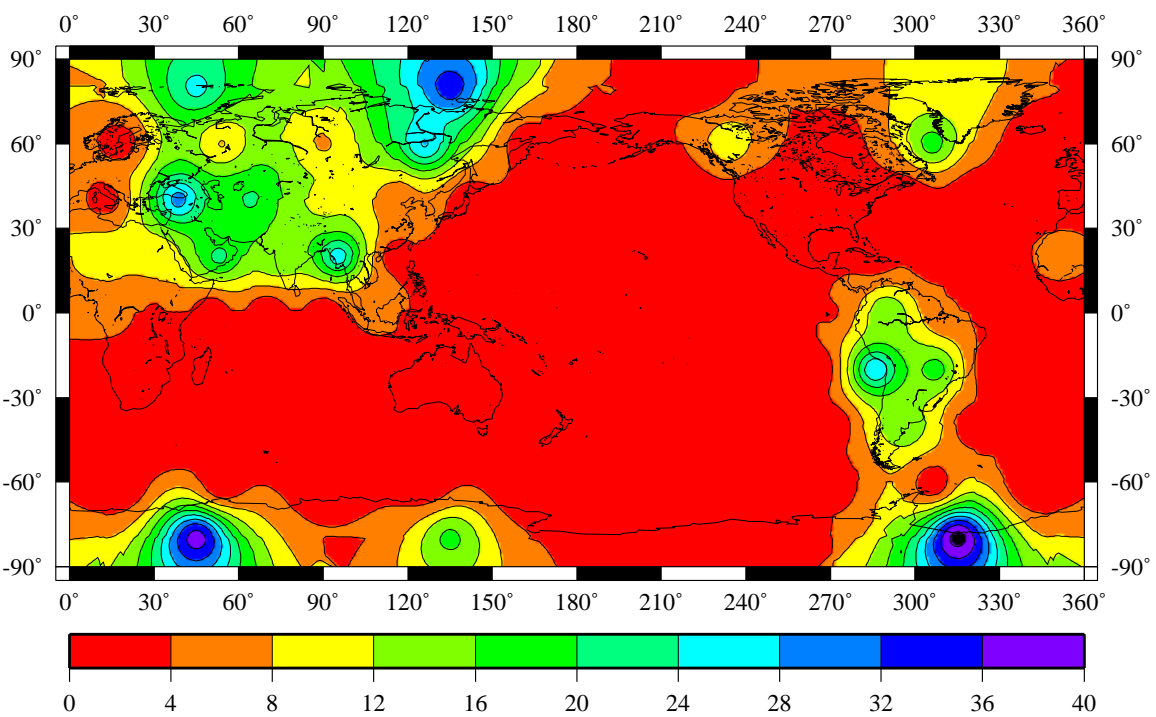

Fig. 5. Scale factors of the error degree variances in the covariance models of the $20^{\circ}$ equal area blocks. and good quality data are used in GPM98A (and OSU91A) the estimated values of $N$ (treated as a free parameter) are equal or very close to the maximum degree of expansion of OSU91A. The scale factor of the error degree variances is very small (below 1) and the RMS value varies between 1 and 2. More specifically, the following points could be notices:

- In the case of the best fitting ( $N$ treated as free parameter) and in the polar caps, the radius of the Bjerhammar sphere is generally very close to the mean radius of the spherical approximation of the Earth.

- In zone 7 (blocks 81 to 94) the estimated values of optimum $N$ are (with the exception of block 92) equal to the maximum degree of expansion of OSU91A. Simoultaneously, the model fits the empirical values very well, and the scale factor for the error degree variances remains very small (below 1). The reason could be that zone 7 is mostly an oceanic area and the data used in GPM98A (and in OSU91A) were coming from altimeter data. Block 92 is a continental block with very poor coverage in gravity values, covering South America (see e.g. ESA, SP-1233, page 17, Fig. 2.3.)

- The same as previously is valid for the also oceanic zone 8, with the exception of blocks 97, 98 and 103.

- The same is valid for the blocks 21 to 28 of zone 3 , which are either oceanic blocks covering parts of Pacific or Atlantic, or continental blocks covering the very well investigated North America.

The values of the variance, of the scale factor and of the depth to the Bjerhammar sphere were plotted in Figs. 3, 4 and 5, for the results of four free parameters. In Fig. 3 the larger values of variance are shown in the mountain chains of Central Asia and Southwest America, while in the oceans the maximum does not exceed $300\left(10 \mu \mathrm{ms}^{-2}\right)^{2}$. One reason for this behavior could be the fact that for the computation of GPM98A good and homogeneous mean gravity anomalies $\left(5^{\prime} \times 5^{\prime}\right.$ means) were used for the oceanic areas, derived from the recent satellite missions (Andersen and Knudsen, 1998). On the other hand the gravity values used in continental mountainous areas are highly correlated with the topography.

A similar pattern is shown in Fig. 4. Large values of scale factors of error degree variances are shown in the same areas. Moreover large values appeared in polar areas, with a poor coverage of measurements, even from satellite missions.

Finally, the plot of the depth to Bjerhammar sphere shows maximum values in only two regions: In the Central south Asia and in the Central Africa.

\section{Conclusion}

For the modelling of GOCE data Least-Squares collocation may be used either to construct a solution based on regionally interpolated data such as $T_{z z}$ on a sphere with a radius equal to the mean distance of GOCE from the Earths centre, or to screen the data for gross errors by predicting the observations from surrounding earlier observed data. The prediction by LSC of the $T_{z z}$ "normal"-point data requires that the regional covariance functions are estimated and subsequently modelled analytically.

To investigate these possibilities a global data set was generated using the GPM98A model complete to degree 1800 , from which OSU91A has been subtracted. The covariance functions were estimated in equal-area blocks of size $22.5^{\circ} \times 22.5^{\circ}$ at Equator, having a $2.5^{\circ}$ overlap. For each block an analytic model was determined based on 4 parameters: the depth to the Bjerhammar sphere (determines correlation), the free-air gravity anomaly variance, a scale factor of the OSU91A error degree-variance and a maximal summation index, $N$, of the error degree-variances. 
Table 1. Fitting of the empirical covariance functions of $20^{\circ}$ equalarea blocs

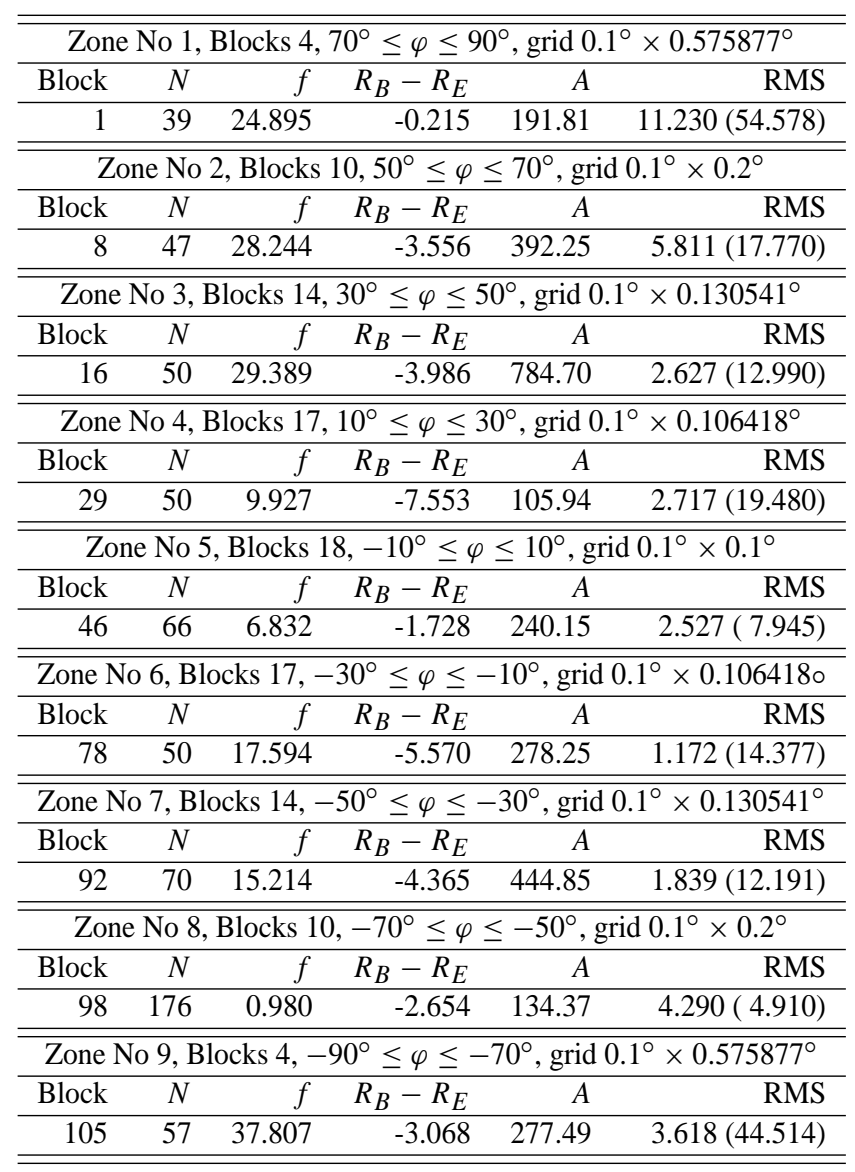

Globally all the parameters varies within wide limits, depending on the quality of the data used to construct OSU91A and GPM98A and the general conditions such as occurrence of mountain chains. The variation of the parameters show that it is necessary to use regional covariance models in order to have a realistic signal to noise ratio and that the co- variance models must be updated when real data becomes available from GOCE.

Acknowledgements. This paper is a contribution to the SAGRADA project (http://www.gfy.ku.dk/ cct/sagrada.htm) sponsored by a grant from the Danish Science Research Council.

\section{References}

Andersen, O. B. and Knudsen, P.: Global gravity field from ERS1 and Geosat geodetic mission altimetry, J. Geophys. Res, 103, (C4), 8129-8137, 1998.

Arabelos, D. and Tscherning, C. C.: Calibration of satellite gradiometer data aided by ground gravity data, Jo G., 72, 617-625, 1998.

ESA,SP-1233 (1): Gravity field and Steady -State Ocean Circulation Mission. Reports for mission selection, The four candidate Earth explorer core missions, 1999.

Jekeli, C.: An investigation of two models for the degree-variances of global covariance functions, Reports of the Department of Geodetic Science, No 275, The Ohio State University, Columbus, Ohio, 1978.

Knudsen, P.: Estimation and modelling of the local empirical covariance functions using gravity and satellite altimeter data, Bull. Géod., 61, 145-160, 1987.

Sansó, F. and Tscherning, C. C.: Fast spherical collocation: A general implementation, IAG Symposia, 125, J. Adám and K.-P. Schwarz (Eds), 131-137, Springer Verlag, 2002.

Tscherning, C. C. and Rapp, R. H.: Closed covariance expressions for gravity anomalies, geoid undulations, and deflections of the vertical implied by anomaly degree-variance models, Reports of the Department of Geodetic Science, No 208, The Ohio State University, Columbus, Ohio, 1974.

Tscherning, C. C., Knudsen, P., and Forsberg, R.: Description of the Gravsoft package, Geophysical Institute, University of Copenhagen, Technical Report, 1994.

Wenzel, H.-G.: Ultra high degree geopotential models GPM98A and GPM98B to degree 1800 Proc, Joint Meeting Int. Gravity Commission and Int. Geoid Commission, Budapest, 10-14 March. Rep 98:4, Finnish Geodetic Institute, Helsinki, 71-80, 1998. 